\title{
Cisplatin fails to induce puma mediated apoptosis in mucosal melanomas
}

\author{
Marie Kristin Fritsche ${ }^{1}$, Veronika Metzler ${ }^{1}$, Karen Becker ${ }^{2}$, Christian Plettenberg $^{3}$, \\ Clemens Heiser ${ }^{1}$, Benedikt Hofauer ${ }^{1}$, Andreas Knopf ${ }^{1}$ \\ ${ }^{1}$ Technische Universität München, Hals-Nasen-Ohrenklinik und Poliklinik, 81675 München, Germany \\ ${ }^{2}$ Universität München, Institut für Allgemeine Pathologie und Pathologische Anatomie, 81675 München, Germany \\ ${ }^{3}$ Heinrich Heine Universität Düsseldorf, Hals-Nasen-Ohrenklinik, 40225 Düsseldorf, Germany
}

Correspondence to:

Marie Kristin Fritsche, e-mail: k.fritsche@Irz.tum.de

Keywords: melanoma, sinonasal, survival, p53, cell-cycle

Received: December 18, $2014 \quad$ Accepted: January 23, $2015 \quad$ Published: March 26, 2015

\begin{abstract}
Objectives

Mucosal melanomas (MM) are aggressive subtypes of common melanomas. It remains unclear whether limitations in their resectability or their distinctive molecular mechanisms are responsible for the aggressive phenotype.
\end{abstract}

Methods

In total, 112 patients with cutaneous melanomas (CM) and 27 patients with MM were included. Clinical parameters were analysed using Chi square, Fisher exact and student's $t$-test. Survival rates were calculated by Kaplan-Meier. Analysis of p53, p21, Mdm2, Hipk2, Gadd45, Puma, Bax, Casp9 and Cdk1 via quantitative PCR and immunohistochemistry (IHC) was performed. TP53 induction after cisplatin treatment was analysed in 10 cell lines (melanocytes, four MM and five CM) using western blot (WB) and qPCR.

Results

The overall/recurrence-free survival differed significantly between MM (40 months and 30 months) and CM (90 months and 107 months; $p<0.001)$. IHC and WB confirmed high p53 expression in all melanomas. Hipk2 and Gadd45 showed significantly higher expressions in CM $(p<0.005 ; p=0.004)$. QPCR and WB of wildtype cell lines demonstrated no differences for p53, p21, Mdm2, Bax and Casp9. WB failed to detect Puma in MM, while Cdk1 regulation occurred exclusively in MM.

\section{Conclusions}

The aggressive phenotype of $M M$ did not appear to be due to differential expressions of p53, p21, Mdm2, Bax or Casp9. A non-functional apoptosis in MM may have further clinical implications. 


\section{INTRODUCTION}

In Europe, 18 of 100,000 people are annually diagnosed with malignant melanoma, and the incidence is continuously increasing. Earlier detection by intensive clinical efforts have led to an enhanced five-year survival rate of $89 \%-94 \%$ [1]. Mucosal melanomas represent an infrequent subtype representing $1 \%$ of the overall cohort [2]. The majority of MM originates in the sinonasal region. Other tumour sites such as the oral cavity, uvea, or the urogenital and gastrointestinal tracts occur infrequently [3]. In contrast to their cutaneous counterparts, MM present a highly aggressive subtype with a poor five-year survival rate of approximately $17 \%$ [4]. The sinonasal region is a difficult site to access surgically, often preventing a radical surgical approach with sufficient R-status. Therefore, locoregional recurrent disease might be a possible explanation for the limited survival. Molecular mechanisms underlying the highly aggressive phenotype remain unclear. While the aetiology of MM is still unclear, molecular changes that may contribute to tumour development in CM are widely discussed [5-10]. While TP53 is mutated in many solid tumours, mutations in CM and MM are rare [11]. However, accumulation of wild-type p53 can be detected in the majority of CM and MM. The mechanisms explaining the p53 protein stabilisation remain unclear. Mutations in p53modifying proteins Mdm2 (mouse double minute 2) and Hipk2 (homeodomain-interacting protein kinase 2) can be excluded [12]. Cellular stress, such as damage induced by UV light and cytotoxic agents, leads to p53 stabilisation by blocking its degradation through $\mathrm{Mdm} 2$ and posttranslational modifications [13]. Once p53 is activated, it functions as a transcription factor in the expression of a broad variety of target genes involved in cell cycle regulation, DNA repair and apoptosis [14]. MDM2 is a direct target gene, which mediates p53 degradation. Under stress conditions, this feedback loop is inhibited by phosphorylation of $\mathrm{p} 53$ on Thr18 through Ck1 (casein kinase 1) thereby disrupting Mdm2-p53 binding [15]. Stabilised and activated p53 can induce cell cycle arrest by enhancing the expression of $\mathrm{p} 21$ which itself inhibits Cdk1 (cyclin dependent kinase 1) and other Cdks, decelerating cell cycle progression [16]. The influence of p53 in DNA repair was explored by inducing the expression of GADD45A (growth arrest and DNA damage-inducible 45). In addition, GADD45A is involved in cell cycle regulation, survival and apoptosis [17]. The p53-induced apoptosis is accomplished by expressing the target genes PUMA (p53 upregulated modulator of apoptosis) and BAX. Through its BH3 domain, Puma binds the anti-apoptotic proteins $\mathrm{Bxl}-\mathrm{X}_{\mathrm{L}}$ and $\mathrm{Bcl}-2$ prohibiting their inhibitory function on Bax [18]. Active Bax permeabilizes the mitochondrial membrane, releasing cytochrome $\mathrm{c}$. This leads to caspase activation resulting in apoptosis [19]. Despite missing TP53 mutations in CM and MM, the in vivo and in vitro response of classical p53-inducing chemotherapeutic agents such as cisplatin is poor [20].
We give a detailed assessment of the clinical characteristics of 139 patients with malignant melanoma of the head and neck, including $112 \mathrm{CM}$ and $27 \mathrm{MM}$. To investigate whether or not the aggressive phenotype in $\mathrm{MM}$ is due to an aberrant p53 pathway we analysed the protein and mRNA expression of p53 and its targets. Formalin-fixed, paraffin-embedded (FFPE) samples of 40 patients with head and neck CM and MM were differentially analysed using immunohistochemistry and real-time (RT-) PCR (qPCR). Furthermore, we investigated the functional integrity of p53 in CM and MM melanoma cell cultures by cisplatin incubation. Experimental data was put into clinical context.

\section{RESULTS}

\section{Epidemiology}

A total of 139 patients with malignant melanoma of the head and neck, treated in the Department of Otorhinolaryngology, Technical University Munich, were included in the current study. There were 112 patients with $\mathrm{CM}$ and 27 patients with MM. Twenty-five patients with MM demonstrated a sinonasal tumour manifestation, and two patients had tumours in the oral cavity. Patients with $\mathrm{CM}$ were significantly younger (mean age of 58 years, SD: 15$)$ than patients with MM (70 years, SD: $12, p<$ $0.001)$. Concordant with the UICC classification system, the majority of MM was classified as T3 tumours. At the time of diagnosis two patients (7\%) with MM showed locoregional lymph node involvement, as compared to 15 patients $(13 \%)$ with $\mathrm{CM}(p<0.396)$. Lymph node involvement in $\mathrm{MM}$ exclusively occurred in oral MM. In contrast, MM tended to exhibit less frequent distant metastases at the time of diagnosis $(p<0.064)$ (Table 1$)$.

\section{Survival analysis}

Recurrent disease was demonstrated in $10(40 \%)$ MM and $30(19 \%)$ CM $(p<0.001)$. The mean diseasefree survival time in $\mathrm{MM}$ was significantly reduced (30 months) when compared with CM (107 months) $(p<$ 0.001). After a mean follow-up of 89 months, the median overall survival (OS) differed significantly between both groups (MM: 40 months; CM: 99 months; $p<0.001$ ) (Figure 1).

\section{p53 mutation status in MM cells and $C M$ cell lines}

Sequence analysis of the DNA-binding domain, comprising exon 5-8, of TP53 revealed no aberration in MM cells (TU-MM1-TU-MM4). The identification of aberrations in CM cell lines (one missense mutation SK-MEL 3: R267W and one frameshift deletion IGR-37: C229DelTG) was previously described [21]. Whereas IGR-37 expresses mutant transcript only, SK-MEL 
Table 1: Epidemiological data of mucosal melanoma and cutaneous melanoma. MM Mucosal melanoma; CM Cutaneous melanoma.

\begin{tabular}{|c|c|c|c|c|}
\hline & All & MuM & $\mathbf{C M}$ & $p$-value \\
\hline$n$ & 139 & 27 & 112 & \\
\hline Age at diagnosis (SD) & $60(15)$ & $70(12)$ & $58(15)$ & $<0.001$ \\
\hline $\operatorname{Sex}(f / m)$ & $65 / 74$ & $16 / 11$ & $49 / 63$ & $=0.126$ \\
\hline $\mathrm{T} 1$ & 24 & 0 & 24 & $<0.032$ \\
\hline $\mathrm{T} 2$ & 22 & 0 & 22 & $<0.012$ \\
\hline $\mathrm{T} 3$ & 25 & 21 & 15 & $<0.001$ \\
\hline $\mathrm{T} 4$ & 20 & 6 & 14 & $<0.198$ \\
\hline Tis & 19 & 0 & 19 & $<0.022$ \\
\hline $\mathrm{Tx}$ & 29 & 0 & 18 & $<0.005$ \\
\hline $\mathrm{N}+$ & 17 & 2 & 15 & $<0.396$ \\
\hline $\mathrm{M}+$ & 13 & 0 & 13 & $<0.064$ \\
\hline
\end{tabular}
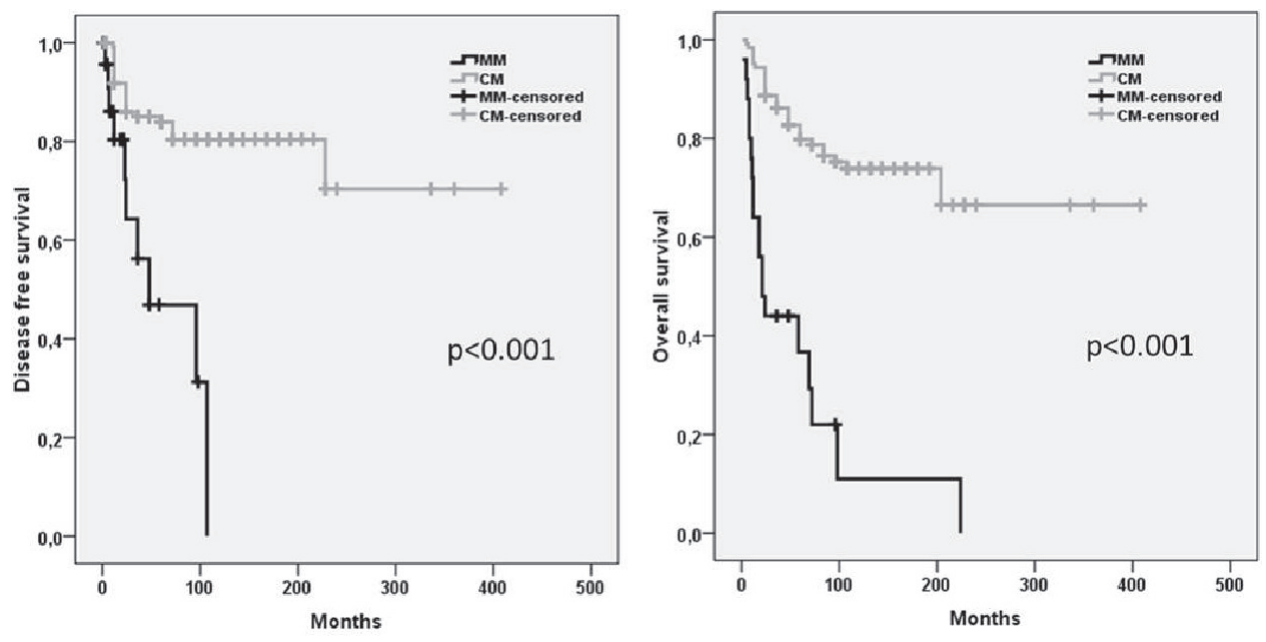

Figure 1: Kaplan-Meier estimates of the disease free and overall survival in patients with MM and CM. MM Mucosal melanoma; CM Cutaneous melanoma.

3 retains $p 53$ wild-type transcript. Sequence analysis revealed wild-type p53 in SK-MEL 30, MEL JUSO and COLO-849 (Data not shown).

\section{Proteins involved in the $\mathbf{p 5 3}$ pathway}

Immunohistochemical staining revealed p53 positivity in $94 \%$ of the $\mathrm{CM}$ and $80 \%$ of $\mathrm{MM}$ with a significantly higher expression in CM. Mdm2 that is directly involved in the p53 protein stabilisation was positive in $71 \%$ of $\mathrm{MM}$ and $81 \%$ of $\mathrm{CM}$ without statistical significance. Immunohistochemical staining revealed a striking difference of Hipk2 staining patterns between the groups $(p<0.005)$. Hipk2, responsible for $\mathrm{p} 53^{\text {Ser46 }}$ phosphorylation therefore mediating enhancement of promoter-specific DNA binding, was negative in $88 \%$ of MM, whereas $44 \%$ of CM showed Hipk 2 positivity. Despite the high number of p53-positive cases, the majority of MM (58\%) and CM (63\%) showed a weak p21 staining. CM demonstrated a significantly increased Gadd45a staining in $100 \%$ of analysed samples. Ninety-two percent of MM stained positive for Gadd $45 \mathrm{a}$ ( $p=0.004)$. Of the CM cases, 94\% stained positive for Puma and $62 \%$ were positive for Bax; however, only $78 \%$ of the MM were positive for Puma and $42 \%$ for Bax. The observed tendency failed to reach statistical significance ( $p=0.08 ; p=0.09)$. In contrast to the high expression of pro-apoptotic proteins, the vast majority of CM (92\%) and MM (81\%) were negative for Casp9. Cell-cycle regulating protein Cdk1 was detected in $38 \%$ of CM and $63 \%$ of MM (Table 2 ). 
Table 2: Immunhistochemical data of proteins involved in the p53 pathway. MM Mucosal melanoma; CM Cutaneous melanoma.

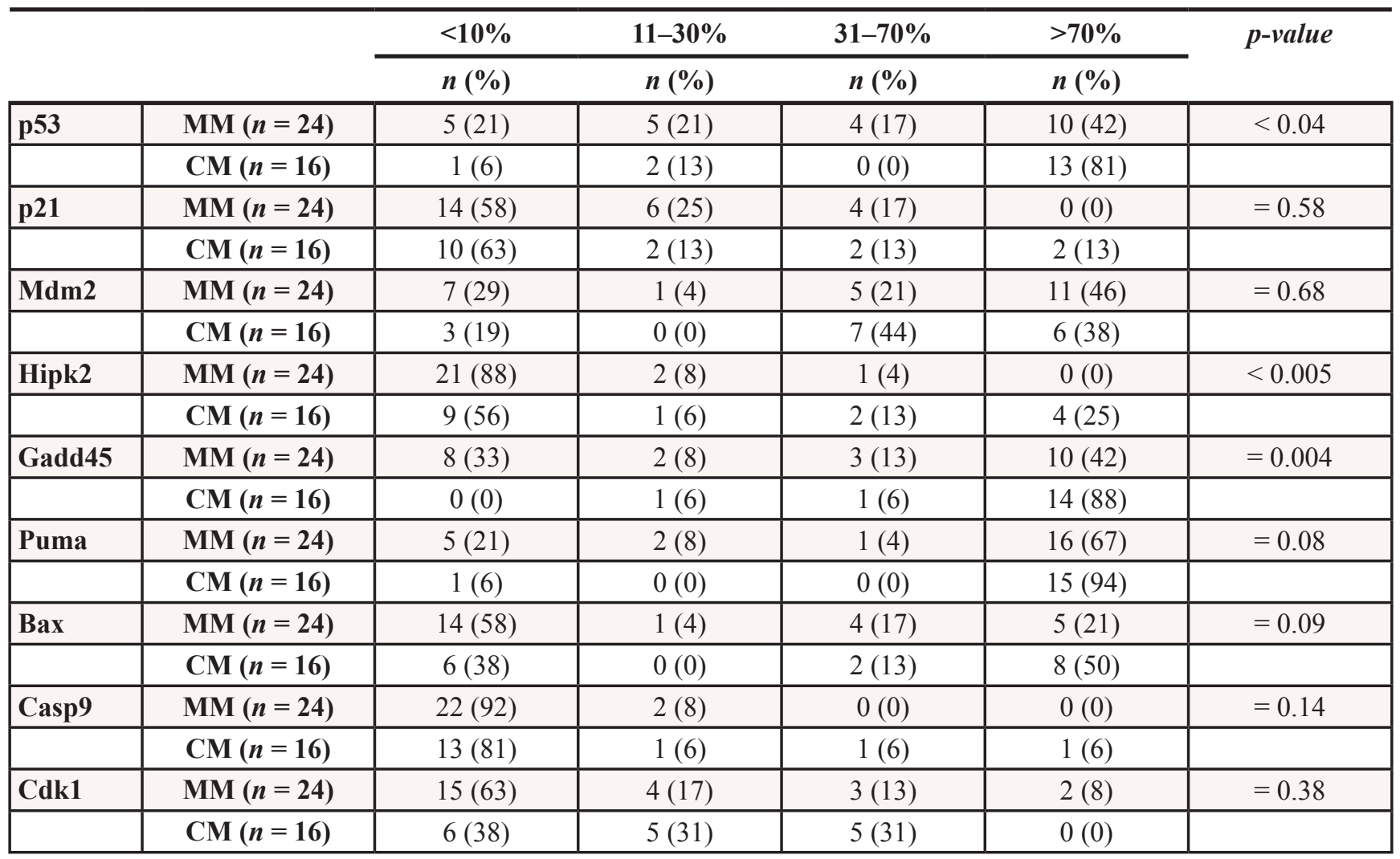

\section{Quantitative PCR of the p53 pathway in FFPE tumour samples}

QPCR of FFPE tumour samples did not show significant differences in the expression of TP53, BBC3, $C A S P 9, B C L 2 A 1$, and $C D K 1$. HIPK2, MDM2, CDKN1A, and $B A X$ mRNA expression was increased in MuM demonstrating differences from 2.6- to 4.2-fold. GADD45 showed 2.4-fold decreased expression in MM (Figure 2).

\section{Quantitative PCR of the p53 pathway in MM cells and $\mathrm{CM}$ cell lines}

QPCR of untreated MM cells and CM cell lines revealed different expression patterns of analysed genes due to the underlying p53 mutation status. Primary MM cells and melanoma cell lines harbouring wild-type p53 demonstrated a significantly lower TP53 mRNA expression when compared with primary melanocytes. The lowest TP53 mRNA levels were determined for p53knockout cell line IGR-37. Parallel with p53 mRNA levels, significantly decreased mRNA levels were detected for $\mathrm{p} 53$ stabilizing HIPK2 in all p53 wild-type cell lines and IGR37. No differences were observed for melanoma cell lines expressing mutated p53. Direct p53 target gene CDKN1A demonstrated significantly lower expressions for all tumour cell lines when compared with primary melanocytes. A significant and stepwise decrease can be observed in MM cells, from melanoma cell lines harbouring wild-type p53 to melanoma cell lines with mutated p53. GADD45A mRNA was expressed equally in primary melanocytes and MM cells; a significantly higher expression than that of CM cell lines. $B B C 3$ and $B A X$ had significantly lower expression in $\mathrm{MM}$ and CM cells. The lowest levels demonstrated p53knockout IGR-37. Analysis of CASP9 demonstrated a significantly decreased mRNA expression when compared with primary melanocytes. No differences were observed for $C D K 1$ expression levels in MM and CM, except IGR37 (Figure 3). To investigate the functional integrity of the p53 downstream, cells were treated with $8 \mu \mathrm{M}$ cisplatin. There was no regulation of TP53 in the analysed cell lines. All cell lines harbouring wild-type p53 demonstrated an upregulation of $M D M 2$, with the highest expression in melanocytes and MM. HIPK2 was downregulated in melanocytes and CM cell lines that expressed mutated p53. Cell cycle regulation $C D K N 1 A$ was upregulated in all cell lines after treatment with cisplatin, while $G A D D 45 A$ was upregulated only in melanocytes. $B B C 3$ mRNA expression increased in CM cell lines with wild-type p53 and in MM. QPCR failed to demonstrate a regulation $B A X$ or $C A S P 9$. A $C D K 1$ downregulation was exclusively seen in $\mathrm{MM}$ (Figure 3). 


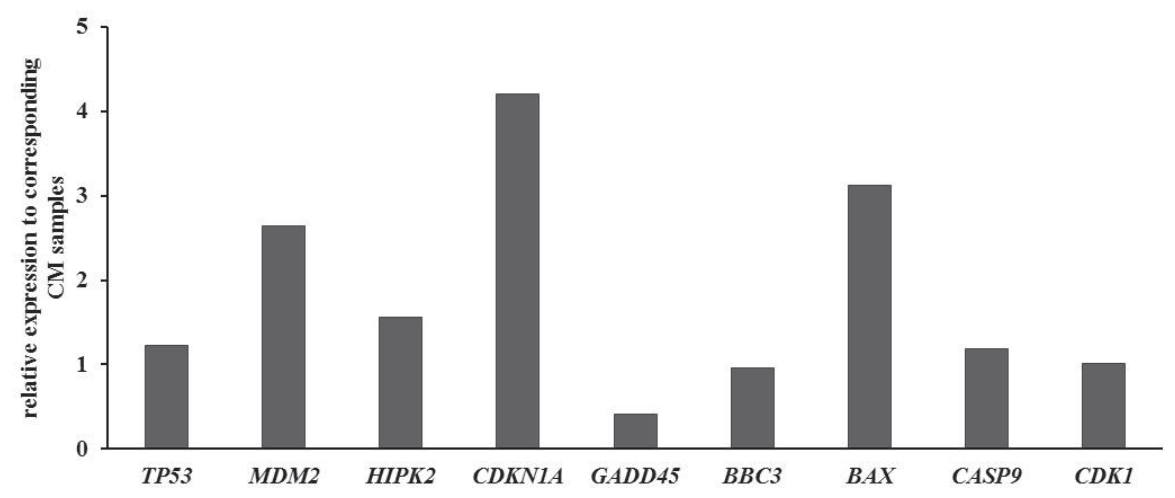

Figure 2: Quantitative PCR of the p53 pathway in FFPE tumor samples. Results were normalized to GAPDH and shown as fold induction compared to CM. MM: Mucosal melanoma; CM Cutaneous melanoma.

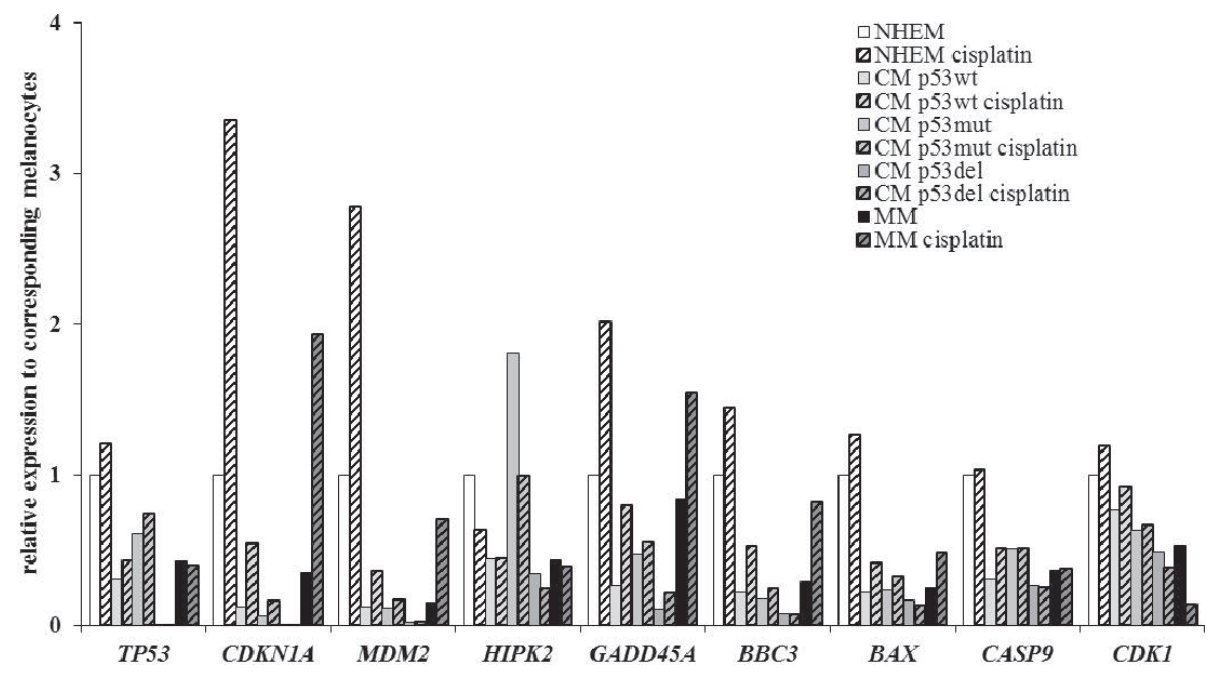

Figure 3: Quantitative PCR of the p53 pathway in primary cells and cell lines without treatment and after $24 \mathrm{~h}$ incubation with $8 \boldsymbol{\mu M}$ cisplatin. Results were normalized to GAPDH and shown as fold induction compared to CM. MM: Mucosal melanoma; CM: Cutaneous melanoma. Cis: Cisplatin.

\section{Western blot of p53 and p53 target genes in cutaneous and mucosal melanoma cell lines}

To further analyse the p53 activation capability after cisplatin treatment on protein level, the protein expression was analysed in western blot experiments. Despite unaltered TP53 mRNA levels, a stabilisation of $\mathrm{p} 53$ protein could be observed in melanocytes and all MM cells. In SK-MEL 30, SK-MEL 3 and IGR-37 cisplatin incubation failed to induce the p53 protein. Cell lines that showed p53 induction demonstrated subsequent $\mathrm{p} 21$ upregulation. Independent of p53 or p21 expression, all cell lines, except SK-MEL30, showed an increased Mdm2 level, even though the protein amount of MM cells was difficult to detect. Treatment with cisplatin failed to induce a consistent response in the p53 downstream signalling. If detectable, Gadd45A and Puma showed no significant regulations. Bax stabilisation was seen in three out of four MM cells and weakly, but not significant, in all CM cells excluding COLO-849. MM and $\mathrm{CM}$ cells tended to regulate the cell-cycle regulating protein
Cdk1 in a different manner. While Cdk1 protein expression is downregulated by cisplatin in MM cells, it is enhanced in CM cells. All untreated cells demonstrated Casp9 expression. Cells did not regulate Casp9 after treatment with cisplatin, nor did they show any cleave products (Figure 4).

\section{DISCUSSION}

MM represents a small subgroup of malignant melanoma. The vast majority of MM originate in the sinonasal region and are clinically aggressive. This study includes 112 patients with $\mathrm{CM}$ and 27 with MM of the head and neck region. The high percentage of MM can be attributed to its sinonasal and oral origin and the subsequent admission to our otorhinolaryngology department. Similar to findings in the current literature, our study showed that patients with MM were significantly older than their cutaneous counterparts (MM 70 years, SD: 12, CM 58 years, SD: 15, $p<0.001$ ) [4]. The prevalence 


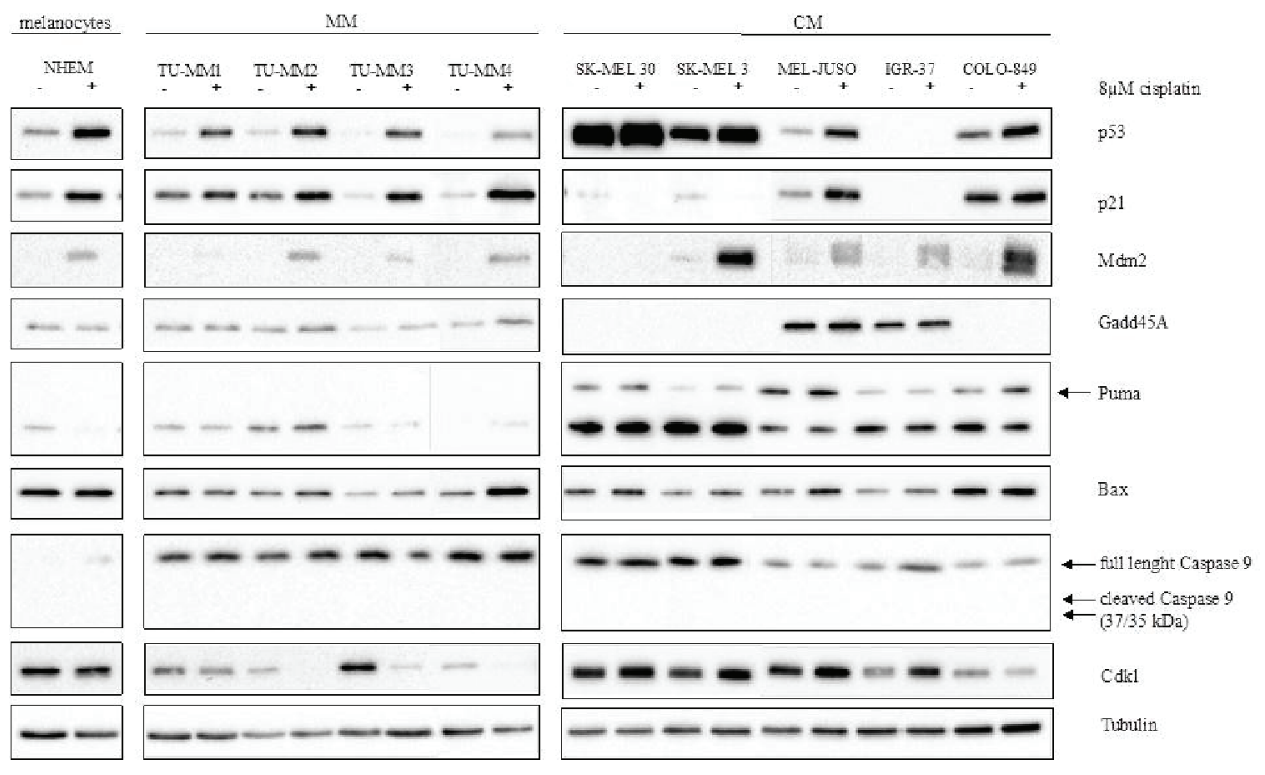

Figure 4: Cisplatin-induction of the p53 pathway in MM and CM cell lines. Protein was isolated from cells treated with either fresh medium or $8 \mu \mathrm{M}$ cisplatin for 24 hours. Tubulin served as loading control. MM: Mucosal melanoma; CM: Cutaneous melanoma. Cis: Cisplatin.

of women was not determined in this study. According to the UICC staging system, most MM were classified as T3 tumours. At time of diagnosis no difference was seen in the locoregional metastasis rate (MM 7\%, CM 13\%, $p<0.396$ ), but MM did demonstrated a tendency for less frequent distant metastases at the time of diagnosis $(p<0.064)$. A significant difference was seen in the disease-free and overall survival in favour of $\mathrm{CM}$ $(p<0.001)$. A multifocal tumor expansion and the difficult anatomic site in the sinonasal system often prevent resections with an appropriate R-status. Therefore, recurrence rates are estimated to be about $50 \%-90 \%$ [22] While systemic therapeutic approaches failed to improve patients' prognoses in $\mathrm{MM}$, it remains unclear whether the aggressive phenotype refers to a limited resectability in the sinonasal region or to distinct biological mechanisms. TP53 is frequently mutated in solid tumours, whereas mutations in $\mathrm{CM}$ and $\mathrm{MM}$ are rare [12]. The mutation load in MM is estimated to be five- to ten-fold smaller than in CM [23]. Although protein stabilising mutations are rare, an accumulation of wild-type p53 can be demonstrated in the majority of CM and MM [24]. Recently, a disruption of cell-cycle regulating proteins, $\mathrm{Bcl}-2$, p53 and p16, were associated with the carcinogenesis of primary oral mucosal melanomas [25]. While wild-type p53 demonstrates a short half-life of approximately 30 minutes, p53 is physiologically stabilised after cellular stresses by a broad variety of posttranslational modifications, including acetylation, phosphorylation and sumoylation, increasing its transcriptional activation, or enhancement of DNA and promoter specific binding [26, 27]. An accumulation of the p53 protein in untreated cells implies a disruption of its functional integrity indicating a p53 mutation or an interaction with viral oncogenes [28]. In the current cohort, all MM cells showed wild-type p53, while two mutations were detected in five CM cell lines. In contrast, western blot analysis demonstrated an accumulation of the p53 protein in all cells, except p53-knockout IGR-37. Immunohistochemical staining of 40 tumour samples confirmed the high p53 expression level in CM and MM. In our cohort, CM (92\%) showed a significantly higher p53 expression level than MM $(72 \% ; p<0.04)$. In the absence of protein stabilising mutations in seven of nine tumour cell lines, other mechanisms have to be considered to explain the abrogation of the p53 pathway, particularly the induction of apoptosis, resulting in the accumulation of wild-type p53. Blagosklonny constitutes a regulatory p53 feedback loop in which the loss of p53 function results in a 'compensatory' p53 upregulation by decreasing its degradation [28]. Therefore, the key point that leads to the disruption of the p53 pathway has to be assessed in our cohort. Recently, we demonstrated a functional p53 upstream in different melanoma cell lines [21]. In the current cohort, western blot experiments revealed cisplatin-induced p53 upregulation in all p53 wild-type cells, except SK-MEL 30. The high p53 base level did not result in further induction. Mdm2 and Hipk2 play a pivotal role in the p53 stabilisation via phosphorylation at serine residues. Overexpression of $M D M 2$ by enhanced protein translation was frequently observed in solid tumours and was associated with tumour progression [29-31]. However, qPCR experiments demonstrated a significantly higher expression of $M d m 2 \mathrm{mRNA}$ expression of primary melanocytes when compared with CM and MM. QPCR analysis of FFPE samples and cells revealed a significantly higher MDM2 mRNA expression in MM when compared with CM. Different qPCR levels did not result in a different protein level in IHC or WB analysis, most likely 
due to posttranslational mechanisms or a TP53independent $\mathrm{Mdm} 2$ regulation. For example, a positive feedback loop is described for p53-induced Caspase2mediated Mdm2 cleavage [32]. In addition, regardless of the TP53 mutation status, Mdm2 mRNA expression was upregulated after cisplatin treatment for CM and MM. Importantly, the Mdm2 up-regulation of IGR-37 suggested TP53 independent mechanisms. The hepatocyte growth factor receptor and the insulin like growth factor 1 receptor were found to regulate $M D M 2$ translation by signalling through PI3K and mTOR. Furthermore, several RNA binding proteins were shown to interfere with TP53 and MDM2 [29]. In contrast, CM with mutated p53 showed a significantly higher HIPK2 mRNA expression compared with wild-type cells. Pointing to an enhanced mutational load in CM, IHC staining of 40 tumour samples validated the higher expression in favour for CM [21, 23]. Cell lines of a different mutational status failed to demonstrate a significant HIPK2 mRNA regulation after cisplatin treatment, which points to regulatory mechanisms on protein levels. In agreement with the current literature, we hypothesized a loss of p53 function in SK-MEL 30 which resulted in an incapability to induce $\mathrm{Mdm} 2, \mathrm{p} 21$ and Gadd45a [27]. This most likely indicates an interaction with viral oncoproteins or cellular proteins that stabilise non-functional wild-type p53 [28]. GADD45A, which is involved in DNA repair and cell cycle regulation, demonstrated inconclusive expression patterns. In FFPE samples, qPCR and IHC detected a significantly higher expression in CM, while cell experiments showed opposite results. Untreated CM cell lines expressed Gadd45a in two of five cases, including p53-knockout IGR-37, suggesting TP53-independent mechanisms. FoxO3a, Egr-1, c-myc and ZBRK have been identified as modulators of Gadd45a expression [29]. CDK1 represents another cell cycle regulation gene that is involved in the $\mathrm{G} 2 / \mathrm{M}$ arrest. Immunohistochemical staining, WB analysis and qPCR of FFPE samples and cells revealed no differences in the constitutional $C D K 1 / \mathrm{Cdk} 1$ expression. Interestingly, after treatment with cisplatin, only MM cells decreased mRNA and protein expression of $C D K 1 / \mathrm{Cdk} 1$, whereas CM cells tended to stabilize $\mathrm{Cdk} 1$ protein and therefore promote the maintenance of cell proliferation. Recently, the influence of epigenetics in cutaneous melanoma gained in importance. It was demonstrated that gene-specific hypermethylation silences genes involved in cell cycle regulation, DNA repair, apoptosis and cell signalling [33]. The potential impact of a distinct $C D K 1$ mediated cell cycle regulation in $\mathrm{MM}$ and $\mathrm{CM}$ remains elusive at this point. Immunohistochemistry of FFPE samples and tumour cells showed that the constitutional p53 expression resulted in a significantly higher $C D K N 1 A$ expression in MM when compared with CM. Different $C D K N 1 A$ mRNA levels did not result in a different constitutional protein expression as observed in IHC and WB. With respect to Blagosklonny's constitute of a compensatory upregulation of wild-type p53 due to functional loss, elevated CDKN1A mRNA levels are congruent with an accumulation of wildtype p53 but do not inevitably refer to a more 'physiological' behaviour, because a disruption of the apoptotic cascade will result in an increased regulatory feedback. TP53 downstream genes $B B C 3, B A X$ and $C A S P 9$ showed no difference in the mRNA expression in FFPE samples and untreated cells, except for a higher $B A X$ expression for MM in FFPE samples. These results were verified on protein level. Immunohistochemical analysis of staining patterns showed no significant differences between the groups. Interestingly, despite a detectable mRNA level in FFPE and cell lines, MM failed to detect Puma in cell lines by WB experiments. Puma expression correlates inversely with the melanoma malignancy grade, and weak Puma expression is associated with poorer overall survival, suggesting Puma as a marker for disease aggressiveness [35, 36]. Recent studies demonstrated the regulation of Puma by oncogenic miRNAs [34]. In human oral squamous carcinoma cells, Puma was a direct target of miR-222 and mediated the diminished apoptosis after treatment with cisplatin [35]. Additionally, Puma degradation in melanoma cells could be inhibited with chloroquine, promoting apoptosis and demonstrating a significant role of Puma in apoptosis of melanoma cells [36]. In support of these results, we were not able to detect an upregulation of $C A S P 9 \mathrm{mRNA}$ or protein after cisplatin treatment. Recently, the impact of Puma was highlighted in $B R A F$ (V600E) melanoma cell lines. Unexpectedly, treatment with $M E K$ inhibitors led to an inhibition of cisplatin-induced apoptosis in some cell lines via activation of the $P I 3 K / A K T$ pathway [37]. The $P I 3 K-A K T$ pathway was demonstrated to contribute to melanoma resistance as well as its tumour initiation [38]. While mutations in $B R A F$ occur infrequently in MM, pAkt and pErk expression were demonstrated in sinonasal MM [9, 39]. Interestingly, primary melanocytes do not regulate pro-apoptotic proteins after cisplatin treatment. This phenomenon is described for many normal cell types, which are intrinsically apoptosis-resistant and results in chemo-resistant tumours after malignant transformation [40]. This may explain why melanoma is resistant to the majority of chemotherapeutics [41]. Furthermore, Apaf-1 inactivation is described for melanoma cells, supporting this hypothesis [42]. However, the disruption of Puma mediated apoptosis in MM may contribute, despite the challenging anatomic location, to the aggressive phenotype. Therapeutic strategies inhibiting the PI3K-Akt$m T O R$ pathway or activating the apoptotic capability of Puma are of major clinical import [39, 43].

\section{CONCLUSIONS}

Our study confirmed the highly aggressive phenotype of MM. Comprehensive analysis of the p53 pathway in MM and CM revealed no differences in TP53 
and direct targets $C D K N 1 A$ and $G A D D 45 A$ in wild-type cells that represent the majority of MM and CM. The regular induction of direct TP53 target genes failed to induce apoptosis mediated by CASP9. A disruption of $B B C 3$ mediated apoptosis may result in the poor response of MM after treatment with cisplatin, and developing therapeutic approaches is of major clinical import. The potential influence of a distinctive $C D K 1$-dependent cell cycle regulation has to be further investigated.

\section{MATERIALS AND METHODS}

\section{Patient selection}

The study included 139 patients with malignant melanoma of the head and neck, including 112 patients with $\mathrm{CM}$ and 27 patients with MM. Tumor samples were obtained from the tissue collection of the Institute of Pathology at the Technical University Munich. The tissue collection was approved by the local ethical committee. Tumor samples were histologically reviewed by at least two experienced pathologists. Clinical parameters and survival data were retrospectively collected including age at diagnosis, sex, TNM-staging, recurrence, death and loss to follow-up. Patients with lacking data, incomplete staging, and refused or unfinished treatment were excluded from survival analysis. The mean followup time was 89 months (range: 0-408 months). Paraffinembedded tumour (FFPE) samples from $16 \mathrm{CM}$ and 24 $\mathrm{MM}$ were randomly selected and analysed with qPCR and immunohistochemistry (IHC).

\section{Statistical analysis}

Differences between both groups were analysed using the Chi square test and Fisher exact test for categorical and the unpaired student's $t$-test for continuous variables. As main endpoints the overall survival (OS) and recurrencefree interval (RFI) were assessed measuring the time from treatment to locoregional recurrence and death, and/or distant metastasis. Survival rates by the log-rank test for univariate analysis with $p$-values $<0.05$ were considered statistically significant (SPSS Inc., Chicago, IL).

\section{Mammalian cell culture and treatment}

Primary cells were obtained from mucosal melanoma tumour tissue. The tumour tissue was cut into little pieces and dried on culture dishes upside down for 30 minutes before medium was added. After three to four days, the tissue was removed and adhered mucosal melanoma cells were cultured. The mucosal melanoma primary cells TUMM1-TU-MM4, as well as the melanoma cell lines SKMEL 30, IGR-37, MEL-JUSO, COLO-849 and SK-MEL 3 (DSMZ, Braunschweig, Germany) were cultured under standard conditions $\left(37^{\circ} \mathrm{C}, 5 \% \mathrm{CO}_{2}\right.$, fully humidified atmosphere) in Dulbecco's Modified Eagle's Medium or Roswell Park Memorial Institute 1640 medium (TUMM3, TU-MM4, SK-MEL3), supplemented with $10 \%$ foetal calf serum (PAA Laboratories, Cölbe, Germany), $1 \%$ penicillin-streptomycin and 1\% glutamine (all from Biochrom, Berlin, Germany). Normal human epidermal melanocytes (NHEM) were cultured in Melanocyte Medium 2 (PromoCell, Heidelberg, Germany). Cells were treated with $8 \mu \mathrm{M}$ cisplatin (Teva, Ulm, Germany) for 24 hours before protein or RNA was isolated.

\section{RNA extraction from cells and FFPE}

Cells were harvested from culture dishes and RNA was prepared using the RNeasy-Kit (Qiagen, Hilden, Germany) according to the manufacturer's instructions. RNA from FFPE was isolated using the High Pure FFPE RNA Micro Kit (Roche, Mannheim, Germany). The RNA concentration and purity was determined with the NanoDrop system (Thermo Scientific, Wilmington, USA).

\section{DNA extraction from cells and p53 sequencing}

Cells were harvested from culture dishes and DNA was isolated using the DNeasy-Kit (Qiagen) according to the manufacturer's instructions. DNA concentration and purity was determined with the NanoDrop system (Thermo Fischer). For sequencing (Eurofins, Ebersberg, Germany) TP53 exons 5-8 were amplified with KAPA SYBR ${ }^{\circledR}$ FAST (Kapa Biosystems, Woburn, United States) using $60 \mathrm{ng}$ DNA. The following TP53 primers were used: exon5 for 5'- atctgttcacttgtgecetg, rev 5'- aaccagccctgt cgtctctc, exon 6 for 5'- agggtccccaggectctgat, rev 5'caccettaacccctcctcce, exon7 for 5'- ccaaggcgcactg gcctcatc, rev 5'- cagaggctggggcacagcagg, exon8 for 5 '- ttccttactgectcttgctt, rev 5'- tgtcctgcttgcttacetcg.

\section{Quantitative real-time PCR}

$250 \mathrm{ng}$ of total RNA was reverse transcribed using the M-MLV reverse transcriptase (Invitrogen, NY, USA) or the QuantiTect Reverse Transcription Kit (Qiagen) for cultured cells or FFPE sections, respectively. QPCR was performed using KAPA SYBR ${ }^{\circledR}$ FAST (Kapa Biosystems) and primers from the QuantiTect Primer Assay (Qiagen). Results were evaluated using the $2^{-\Delta \Delta \mathrm{CT}}$ method. A fold difference equal or larger than 2 was estimated to be statistically significant.

\section{Protein extraction and western blotting experiments}

Total protein extraction was performed using cell lysis buffer (Cell Signaling Technology, Frankfurt, Germany) according to the manufacturer's instructions. The protein concentration in the supernatant was determined with a Bradford assay. Western blotting was performed using $15 \mu \mathrm{g}$ protein per sample, which were 
separated by SDS-polyacrylamide gel electrophoresis and transferred on a polyvinylidene fluoride membrane (Carl Roth, Karlsruhe, Germany) by electroblotting. Antibodies were incubated in 5\% non-fat milk in TBST $(0.1 \%$ Tween-20, $20 \mathrm{mM}$ Tris, $140 \mathrm{mM} \mathrm{NaCl}$, pH 7.6) overnight at $4{ }^{\circ} \mathrm{C}$. Proteins were detected with antibodies against $\mathrm{p} 53$ (DO-7, Dako Deutschland GmbH, Hamburg, Germany), p21 Waf1/Cip1 (12D1), Puma (D30C10), Gadd45a (D17E8), Bax (D2E11), CDK1 (8G10) (Cell Signaling Technology, Danvers MA, United States) and Mdm2 (SMP14, Santa Cruz, Heidelberg, Germany) using the biotechnology SuperSignal West Pico Chemiluminescent Substrate (Thermo Fisher, Rockford IL, USA).

\section{Immunohistochemistry}

FFPE tumour sections $(2.5-3 \mu \mathrm{m})$ were stained with antibodies against p53 (DO-7, Dako), p21 (9L524), GADD45A, HIPK2, MDM2 (US Biological, Massachusetts, USA), CDK1 (EPR165), PUMA (EP512Y), BAX (E63) (Epitomics, Burlingame, CA, USA) and visualized with the Bond Polymer Refine Detection Kit (Leica, Nussloch, Germany). A positive staining was defined as greater than $10 \%$ stained cells. Tissues with known expression of the respective antigens were used as positive controls.

\section{FUNDING}

Authors did not receive any third party funding.

\section{FINANCIAL DISCLOSURE}

All authors state no financial disclosures.

\section{CONFLICTS OF INTEREST}

All authors state no conflict of interest.

\section{REFERENCES}

1. Godar DE. Worldwide increasing incidences of cutaneous malignant melanoma. J Skin Cancer. 2011; 2011:858425.

2. Lourenço SV, Bologna SB, Hsieh R, Sangueza M, Fernandes JD, Nico MM. Establishment and characterization of an oral mucosal melanoma cell line (MEMO) derived from a longstanding primary oral melanoma. Am J Dermatopathol. 2013; 35:248-251

3. Gavriel H, McArthur G, Sizeland A, Henderson M. Review: mucosal melanoma of the head and neck. Melanoma Res. 2011; 21:257-266.

4. Lengyel E, Gilde K, Remenár E, Esik O. Malignant mucosal melanoma of the head and neck. Pathol Oncol Res. 2003; 9:7-12.
5. Davies H, Bignell GR, Cox C, Stephens P, Edkins S, Clegg S, Teague J, Woffendin H, Garnett MJ, Bottomley W, Davis N, Dicks E, Ewing R, Floyd Y, Gray K, Hall S, et al. Mutations of the BRAF gene in human cancer. Nature. 2002; 417:949-954.

6. Edwards RH, Ward MR, Wu H, Medina CA, Brose MS, Volpe P, Nussen-Lee S, Haupt HM, Martin AM, Herlyn M, Lessin SR, Weber BL. Absence of BRAF mutations in UV-protected mucosal melanomas. J Med Genet. 2004; 41:270-272.

7. Haluska FG, Tsao H, Wu H, Haluska FS, Lazar A, Goel V. Genetic alterations in signaling pathways in melanoma. Clin Cancer Res. 2006; 12:2301s-2307s.

8. Chin L. The genetics of malignant melanoma: lessons from mouse and man. Nat Rev Cancer. 2003; 3:559-570.

9. Curtin JA, Fridlyand J, Kageshita T, Patel HN, Busam KJ, Kutzner H, Cho KH, Aiba S, Bröcker EB, LeBoit PE, Pinkel D, Bastian BC. Distinct sets of genetic alterations in melanoma. N Engl J Med. 2005; 353:2135-2147.

10. Vidwans SJ, Flaherty KT, Fisher DE, Tenenbaum JM, Travers MD, Shrager J. A melanoma molecular disease model. PLoS One. 2011; 6:e18257.

11. Kandoth C, McLellan MD, Vandin F, Ye K, Niu B, Lu C, Xie M, Zhang Q, McMichael JF, Wyczalkowski MA, Leiserson MD, Miller CA, Welch JS, Walter MJ, Wendl MC, Ley TJ, et al. Mutational landscape and significance across 12 major cancer types. Nature. 2013; 502:333-339.

12. Gwosdz C, Scheckenbach K, Lieven O, Reifenberger J, Knopf A, Bier H, Balz V. Comprehensive analysis of the p53 status in mucosal and cutaneous melanomas. Int $\mathrm{J}$ Cancer. 2006; 118:577-582.

13. Brooks $\mathrm{CL}, \mathrm{Gu}$ W. New insights into p53 activation. Cell Res. 2010; 20:614-621.

14. Wei CL, Wu Q, Vega VB, Chiu KP, Ng P, Zhang T, Shahab A, Yong HC, Fu Y, Weng Z, Liu J, Zhao XD, Chew JL, Lee YL, Kuznetsov VA, Sung WK, et al. A global map of p53 transcription-factor binding sites in the human genome. Cell. 2006; 124:207-219.

15. Dumaz N, Milne DM, Meek DW. Protein kinase CK1 is a p53-threonine 18 kinase which requires prior phosphorylation of serine 15. FEBS Lett. 1999; 463:312-316.

16. Pellegata NS, Antoniono RJ, Redpath JL, Stanbridge EJ. DNA damage and p53-mediated cell cycle arrest: a reevaluation. Proc Natl Acad Sci U S A. 1996; 93:15209-15214.

17. Tront JS, Hoffman B, Liebermann DA. Gadd45a suppresses Ras-driven mammary tumorigenesis by activation of c-Jun NH2-terminal kinase and p38 stress signaling resulting in apoptosis and senescence. Cancer Res. 2006; 66:8448-8454.

18. Wang $\mathrm{P}, \mathrm{Yu} \mathrm{J}$, Zhang L. The nuclear function of p53 is required for PUMA-mediated apoptosis induced by DNA damage. Proc Natl Acad Sci U S A. 2007; 104:4054-4059. 
19. Chipuk JE, Kuwana T, Bouchier-Hayes L, Droin NM, Newmeyer DD, Schuler M, Green DR. Direct activation of Bax by p53 mediates mitochondrial membrane permeabilization and apoptosis. Science. 2004; 303:1010-1014.

20. Rosenberg SA, Yang JC, Schwartzentruber DJ, Hwu P, Marincola FM, Topalian SL, Seipp CA, Einhorn JH, White DE, Steinberg SM. Prospective randomized trial of the treatment of patients with metastatic melanoma using chemotherapy with cisplatin, dacarbazine, and tamoxifen alone or in combination with interleukin-2 and interferon alfa-2b. J Clin Oncol. 1999; 17:968-975.

21. Knopf A, Plettenberg C, Pickhard A, Bas M, Reifenberger J, Bier H, Balz V. Analysis of the functional integrity of the p53 tumor-suppressor gene in malignant melanoma. Melanoma Res. 2011; 21:380-388.

22. Lee SP, Shimizu KT, Tran LM, Juillard G, Calcaterra TC. Mucosal melanoma of the head and neck: the impact of local control on survival. Laryngoscope. 1994; 104: 121-126.

23. Furney SJ, Turajlic S, Stamp G, Nohadani M, Carlisle A, Thomas JM, Hayes A, Strauss D, Gore M, van den Oord J, Larkin J, Marais R. Genome sequencing of mucosal melanomas reveals that they are driven by distinct mechanisms from cutaneous melanoma. J Pathol. 2013; 230: 261-269.

24. Avery-Kiejda KA, Bowden NA, Croft AJ, Scurr LL, Kairupan CF, Ashton KA, Talseth-Palmer BA, Rizos H, Zhang XD, Scott RJ, Hersey P. P53 in human melanoma fails to regulate target genes associated with apoptosis and the cell cycle and may contribute to proliferation. BMC Cancer. 2011; 11:203.

25. Prasad ML, Patel SG, Shah JP, Hoshaw-Woodard S, Busam KJ. Prognostic significance of regulators of cell cycle and apoptosis, p16(INK4a), p53, and bcl-2 in primary mucosal melanomas of the head and neck. Head Neck Pathol. 2012; 6:184-190.

26. Oren M. Regulation of the $\mathrm{p} 53$ tumor suppressor protein. J Biol Chem. 1999; 274:36031-36034.

27. Gu B, Zhu WG. Surf the post-translational modification network of p53 regulation. Int J Boil Sci. 2012; 8:672-684.

28. Blagosklonny MV. Loss of function and p53 protein stabilization. Oncogene. 1997; 15:1889-1893.

29. Zhang J, Chen X. Posttranscriptional regulation of p53 and its targets by RNA-binding proteins. Curr Mol Med. 2008; 8:845-849.

30. VanderBorght A, Valckx A, Van Dun J, Grand-Perret T, De Schepper S, Vialard J, Janicot M, Arts J. Effect of an hdm-2 antagonist peptide inhibitor on cell cycle progression in p53-deficient H1299 human lung carcinoma cells. Oncogene. 2006; 25:6672-6677.

31. Poremba C, Yandell DW, Metze D, Kamanabrou D, Bocker W, Dockhorn-Dworniczak B. Immunohistochemical detection of p53 in melanomas with rare p53 gene mutations is associated with mdm-2 overexpression. Oncol Res. 1995; 7:331-339.

32. Oliver TG, Meylan E, Chang GP, Xue W, Burke JR, Humpton TJ, Hubbard D, Bhutkar A, Jacks T. Caspase-2mediated cleavage of Mdm2 creates a $\mathrm{p} 53$-induced positive feedback loop. Mol Cell. 2011; 43:57-71.

33. Bode AM, Dong Z. Post-translational modification of $\mathrm{p} 53$ in tumorigenesis. Nat Rev Cancer. 2004; 4:793-805.

34. Skaftnesmo KO, Prestegarden L, Micklem DR, Lorens JB. MicroRNAs in tumorigenesis. Curr Pharm Biotechnol. 2007; 8:320-325.

35. Jiang F, Zhao W, Zhou L, Liu Z, Li W, Yu D. MiR-222 Targeted PUMA to Improve Sensitization of UM1 Cells to Cisplatin. Int J Mol Sci. 2014; 15:22128-22141.

36. Lakhter AJ, Sahu RP, Sun Y, Kaufmann WK, Androphy EJ, Travers JB, Naidu SR. Chloroquine promotes apoptosis in melanoma cells by inhibiting BH3 domain-mediated PUMA degradation. J Invest Dermatol. 2013; 133:2247-2254.

37. Haydn JM, Hufnagel A, Grimm J, Maurus K, Schartl M, Meierjohann S. The MAPK pathway as an apoptosis enhancer in melanoma. Oncotarget. 2014; 5:5040-5053.

38. Davies MA. The role of the PI3K-AKT pathway in melanoma. Cancer J. 2012; 18:142-147.

39. Turri-Zanoni M, Medicina D, Lombardi D, Ungari M, Balzarini P, Rossini C, Pellegrini W, Battaglia P, Capella C, Castelnuovo P, Palmedo G, Facchetti F, Kutzner H, Nicolai P, Vermi W. Sinonasal mucosal melanoma: Molecular profile and therapeutic implications from a series of 32 cases. Head Neck. 2013; 35:1066-1077.

40. Blagosklonny MV. Paradox of Bcl-2 (and p53): why may apoptosis-regulating proteins be irrelevant to cell death? BioEssays. 2001; 23:947-953.

41. Karst AM, Dai DL, Martinka M, Li G. PUMA expression is significantly reduced in human cutaneous melanomas. Oncogene. 2005; 24:1111-1116.

42. Soengas MS, Capodieci P, Polsky D, Mora J, Esteller M, Opitz-Araya X, McCombie R, Herman JG, Gerald WL, Lazebnik YA, Cordon-Cardo C, Lowe SW. Inactivation of the apoptosis effector Apaf-1 in malignant melanoma. Nature. 2001; 409:207-211.

43. Amaravadi RK. PUMA: a puzzle piece in chloroquine's antimelanoma activity. J Invest Dermatol. 2013; 133:2133-2135. 